www. revis tad y o. com

\title{
Influencia del número de artículos y nivel de utilización en el rendimiento de estrategias de resolución para el problema de secuenciación del lote económico
}

On the influence of number of items and utilization on the performance of solution strategies for the economic lot scheduling problem

Raúl Cortés-Fibla, Pilar I. Vidal-Carreras, José P. García-Sabater

Dpto. de Organización de Empresas. Universidad Politécnica de Valencia. Camino de la Vera s/n, Valencia, Spain

racorfi@omp.upv.es, pivicar@omp.upv.es, jpgarcia@omp.upv.es

Fecha de recepción: 28-10-2014

Fecha de aceptación: 10-04-2015

\begin{abstract}
This study analyzes the behavior of various production strategies for the economic lot scheduling problem (ELSP), depending on the context of complexity and uncertainty in which they are used. The performance of various heuristics designed for the classical ELSP problem is analyzed. The influence of number of items and utilization on total cost under different scenarios is considered. The simulation results show a direct dependence of the production environment on the performance of the strategies, and thus their suitability to that particular environment. It is also proven that the variation in performance does not follow the same pattern for each strategy, which reinforces the significance of this study in the process of designing a production strategy for the ELSP.
\end{abstract}

Keywords:ESLP, Complexity, Uncertainty, Simulation, Scheduling;

Resumen: Este trabajo analiza el comportamiento de varias estrategias de producción para el problema de secuenciación del lote económico, ELSP, en función del entorno de complejidad e incertidumbre en el que se emplean. Se estudia el rendimiento de varias heurísticas diseñadas para el problema ELSP clásico, analizando el comportamiento del coste total bajo diferentes escenarios en función del número de artículos y el nivel de utilización. Los resultados del estudio de simulación indican que existe una clara dependencia del rendimiento de las estrategias, y por tanto de la idoneidad de su uso, en relación con el entorno productivo. Se demuestra también que la variación del rendimiento no sigue el mismo patrón para cada estrategia, lo que refuerza la importancia de este estudio en el proceso de diseño de estrategias de producción para el ELSP.

Palabras clave:ELSP, Complejidad, Incertidumbre, Simulación, Secuenciación;

\section{Introducción}

El problema de programación de lote económico, conocido en la literatura por su acrónimo en inglés ELSP (Economic Lot Scheduling Problem) fue definido formalmente por primera vez en Bomberger (1966). Consiste en la programación de la producción de varios productos diferentes $\mathrm{i}=1$..g en una única máquina, en la que un solo producto puede ser producido en cada momento, con el objetivo de minimizar los costes derivados de la gestión del inventario. Desde entonces, se han planteado numerosas variaciones sobre las condiciones del problema original con el objetivo de adaptar mejor la definición del problema a la realidad industrial. En (Chan et al. 2013) se presenta una revisión reciente de las diferentes variantes del problema estudiadas hasta la fecha. En particular, el problema que consideramos en este estudio tiene las siguientes características:

- Los ratios de producción de cada artículo $p_{i}$ son deterministas y constantes

- $\quad$ Los ratios de demanda (con media $d_{i}$ ) son estocásticos pero estacionarios 
Los costes $A_{i}$ y tiempos $c_{i}$ de setup, son independientes de la secuencia de producción

- Los costes de inventario $h$ son directamente proporcionales a los niveles de stock $I_{i}$

- $\quad$ Se considera la existencia de retrasos y backorders (ordenes pendientes de servir) cuando no es posible satisfacer la demanda a tiempo

La existencia de backorders implica el compromiso de suministrar la demanda que queda pendiente por falta de inventario en el momento que surge, que se acumula como cantidad pendiente de suministrar. En el momento que hay inventario disponible, se suministra en primer lugar esta cantidad pendiente.

La solución al problema ELSP implica abordar dos decisiones críticas. Por un lado, el criterio de lotificación, es decir, determinar cuál va a ser la cantidad a producir de cada artículo para minimizar los costes totales. Por otro lado, la política de secuenciación, es decir, el criterio de decisión respecto al cual se va a decidir en qué momento lanzar un lote de un artículo.

El enfoque más sencillo para resolver el problema de lotificación del ELSP, es conocido como solución independiente (IS), que emplea el lote económico de pedido (EOQ) según su definición en (Harris 1913), para determinar el tamaño de lote de cada artículo. Por definición, este enfoque representa una cota inferior de costes, al emplear el lote óptimo para cada artículo. Este enfoque ignora cualquier condición de factibilidad, por lo que es frecuente encontrar situaciones en las que la solución óptima mediante este enfoque no es factible. Otros enfoques clásicos para la resolución de este problema de lotificación son: ciclo común (CC) (Hanssmann 1962), periodo básico (BP) (Bomberger 1966), y variación del tamaño de lote (TVL) (Dobson 1987).

Con respecto a la secuenciación, existen numerosos estudios que abordan el problema, como el de (Vergin \& Lee 1978) que hemos empleado en nuestro estudio de simulación, o (Leachman \& Gascon 1988; Segerstedt 1999). Algunos interesantes trabajos presentados más recientemente, son (Kou \& Yang 2009; Löhndorf et al. 2014; Levén \& Segerstedt 2007).

De acuerdo con (Winands et al. 2011), la mayoría de los estudios se centran en la evaluación u optimización de una clase de estrategias de resolución, sin realizar un análisis comparativo con respecto a otras. En este sentido, las conclusiones de un trabajo deben ser consideradas únicamente en el marco de los datos de partida que se han utilizado en el análisis. En este trabajo pretendemos profundizar en esta cuestión. Nuestro planteamiento es que el rendimiento de una determinada estrategia de producción es función del grado de complejidad e incertidumbre del entorno productivo, es decir, de las características concretas de los datos de partida bajo las cuales es empleada. Utilizamos aquí el término complejidad en relación con la complejidad estructural del problema, entendida como la dificultad para alcanzar e implementar una solución factible para una situación concreta del problema, en relación con los datos de partida (Frizelle 1996). En este sentido, se trata de un concepto diferente a la complejidad algorítmica del problema ELSP, que es NP-Hard (Hsu 1983). Para la variante del problema definida, que denominaremos problema marco, existen diversos factores con influencia sobre la complejidad. Es conocido que, ante una situación estable del resto de parámetros del problema, trabajar con un mayor $n^{\circ}$ de artículos añade complejidad al problema (Vaughan 2007), al igual que ocurre con el nivel de utilización (Bomberger 1966; Soman et al. 2006). Por tanto, consideraremos el $n^{\circ}$ de artículos y el nivel de utilización como factores inductores de complejidad, a los que llamaremos drivers de complejidad, que tienen un efecto directo sobre los costes para un mismo problema.

Con respecto a la incertidumbre en los datos, algunos autores (Sox et al. 1999), ya han planteado su efecto sobre los resultados de una determinada estrategia de producción. Es habitual que en situaciones reales, exista incertidumbre o estocasticidad respecto al comportamiento de los datos a lo largo del horizonte de planificación. Se ha analizado, por ejemplo, el efecto de la estocasticidad en la demanda (Brander \& Forsberg 2006), en el ratio de productos defectuosos (Yao \& Huang 2005), y en el tiempo y coste de setup (Allahverdi et al. 2008). En este trabajo consideramos el comportamiento estocástico de la demanda como factor inductor o driver de incertidumbre.

Este estudio pretende analizar el comportamiento de los costes totales de cinco estrategias de producción en relación con los costes del modelo teórico. La hipótesis planteada es que, para cada estrategia, esa relación depende del grado de complejidad y de la incertidumbre en la información de partida. Conocer en profundidad esta relación podría servir, por un lado, para introducir nuevos elementos de análisis al diseñar los algoritmos de control adecuados para cada versión particular del ELSP. Por otra parte, permitiría predecir el comportamiento de una determinada estrategia de producción, y por tanto determinar su grado de idoneidad, para la situación concreta en la que fuera a ser utilizada.

La estructura del trabajo es la siguiente: en la sección 
2 se describen las 5 estrategias de producción analizadas en el estudio de simulación; en la sección 3 se describe el modelo de simulación así como los factores experimentales; en la sección 4 se presenta el análisis y discusión de los resultados; por último en el apartado 5 se describen las principales conclusiones y posibles líneas de trabajo futuro.

\section{Descripción de los métodos para lotificación y secuenciación}

En nuestro estudio de simulación hemos evaluado el comportamiento de cinco estrategias de producción. Para su descripción, utilizamos el criterio de clasificación introducido por Winands et al. (2011) que clasifica las diferentes estrategias en función del enfoque de las dos decisiones críticas en la resolución del ELSP: la política de secuenciación y la política de lotificación.

En primer lugar, hemos trabajado con dos heurísticas clásicas, basadas en dos reglas extensamente empleadas en el problema de gestión del lote económico por punto de pedido; la regla $(\mathrm{s}, \mathrm{Q})$ que denominamos heurística 1 y la regla $(\mathrm{s}, \mathrm{S})$ que denominamos heurística 2. En ambos casos, siempre que el stock de un artículo alcanza su punto de pedido s, el lote del artículo está listo para ser lanzado tan pronto como la máquina quede libre. Si varios artículos alcanzan su punto de pedido antes de que la máquina quede libre, se lanzará en primer lugar el artículo con menor ratio de cobertura tal como se define en [1].

$$
R O_{i}=\frac{I_{i}}{d_{i}}
$$

La única diferencia entre estas dos reglas clásicas, es el hecho de que en la primera el lote lanzado es fijo $(Q)$, al contrario que en la segunda, en el que el lote se calcula como la diferencia entre el stock del artículo en el momento de lanzamiento, y el nivel de stock de referencia, order up to level, (S). Con ello, se pretende verificar si esta diferencia tiene efecto en el rendimiento de la estrategia de producción.

Para cada una de estas dos reglas trabajamos con dos variantes diferentes en cuanto al criterio de lotificación. La variante a emplea un criterio basado en el enfoque IS y la variante b utiliza el enfoque de CC. De acuerdo con esta definición, las cuatro primeras estrategias, que llamamos $1 \mathrm{a}, 1 \mathrm{~b}, 2 \mathrm{a}, 2 \mathrm{~b}$, utilizan una política lotificación local; es decir, la decisión respecto al tamaño del lote sólo considera el nivel de inventario del artículo que es lanzado.

Por último, hemos trabajado con una quinta estrategia de producción, basada en la regla de Magee (Magee \& Boodman 1967), en su variante A, descrita en (Vergin \& Lee 1978), que emplea un criterio de lanzamiento y lotificación que tiene en cuenta el stock del resto de artículos a la hora de interrumpir un lote. Esta última estrategia que denominamos $3 a$, utiliza por tanto una política de lotificación global, teniendo en cuenta el inventario del conjunto de artículos. Respecto a la secuencia de producción, las cinco estrategias utilizan una secuencia de producción dinámica; es decir, la secuencia en la que los elementos se producen cambia de acuerdo a las condiciones específicas de cada instante.

Tabla 1. Estrategias de Producción

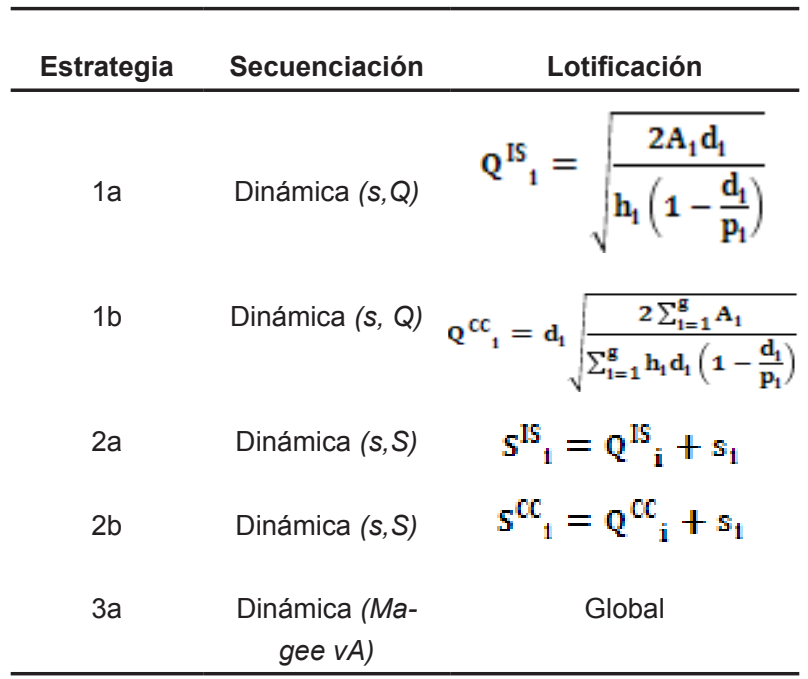

La Tabla 1 resume las características de las estrategias de producción. Para todas ellas, el stock inicial de cada artículo, ' $O_{i}$, se ha definido de modo que en una situación de demanda determinista, cada artículo alcanza su stock de reorden inmediatamente después de que la máquina ha terminado de procesar el elemento anterior en la lista, de acuerdo con [2].

$$
I_{\mathbf{o}_{i}}=\sum_{n=1}^{i}\left(c_{n}+\frac{d_{n} T_{n}}{p_{n}}\right) d_{n}
$$

Así, bajo demanda determinista, la máquina produce consecutivamente los lotes de los elementos sin tiempos de espera entre ellos, y luego espera a que el primer elemento de la lista alcance de nuevo su punto de pedido. Obviamente, en simulaciones con la demanda estocástica, este lanzamiento "sincronizado" no se 
reproduce exactamente. El punto de pedido si se ha establecido como el stock mínimo necesario para satisfacer la demanda durante el tiempo necesario para llevar a cabo el setup y producir el lote correspondiente bajo demanda determinista, de acuerdo con [3]:

$$
s_{i}=\left(c_{i}+\frac{d_{i} T_{i}}{p_{i}}\right) d_{1}
$$

\section{Descripción del modelo de simulación y factores experimentales}

Para evaluar el rendimiento de las heurísticas descritas, se ha desarrollado un modelo de simulación de eventos discretos mediante el software de simulación SIMIO. La arquitectura del modelo está constituida mediante la estructura más simple: Source-Server-Sink. En el servidor (server), se han implementado los algoritmos correspondientes a las estrategias definidas en la sección 2. En base a estos algoritmos, es el servidor quien toma tanto la decisión de secuenciación, es decir, cuál debe ser el siguiente lote a liberar, como la de lotificación, es decir, cuál debe ser el tamaño de dicho lote.

\subsection{Factores experimentales}

Partiendo del problema básico descrito en (Bomberger 1966), se han construido 96 conjuntos diferentes de datos para del mismo problema marco. Para ello, se han construido 4 versiones con diferente $n^{\circ}$ total de artículos, g=10, 20, 30 y 40 artículos, y cuatro niveles de utilización: 0.22, 0.44, 0.66, 0.88 (sin considerar tiempos de setup). Estas 16 combinaciones respecto a $\mathrm{n}^{\circ}$ de artículos y utilización, han sido analizadas bajo 6 niveles de incertidumbre en la demanda, considerando diferentes coeficientes de variación, desde 0 hasta 0.5 con intervalos de 0.1 , haciendo el total de 96 problemas analizados en el estudio.

Para construir los problemas con diferente número total de artículos, se ha tratado deliberadamente de mantener la misma estructura del problema del dataset de Bomberger con $\mathrm{g}=10$, manteniendo los mismos ratios de demanda y de producción, los mismo tiempos de setup y los mismos costes de almacenamiento. Para ello, decidimos multiplicar el número de artículos por 1, 2, 3 y 4 partiendo del problema de 10 artículos, y reduciendo la demanda de cada artículo por el mismo factor. Por otro lado, los 4 niveles de utilización, al igual que en (Bomberger 1966), se obtienen multiplicando el ratio de demanda por un factor $1,2,3$, y 4 en cada caso obteniendo así los niveles de $0.22,0.44,0.66$ y 0.88 .
Respecto a la definición de un stock de seguridad, puesto que el proceso de simulación permite determinar exactamente el nivel máximo de rotura de stock que alcanza cada artículo tras el experimento, se ha empleado esta cantidad como stock de seguridad necesario. Mediante este procedimiento, el stock de seguridad se convierte en un factor de coste determinante que refleja la influencia del número de artículos, nivel de utilización e incertidumbre en la demanda, sobre el rendimiento de cada estrategia de producción.

\subsection{Dinámica de la simulación}

Del mismo modo que en (Brander et al. 2005) todas las simulaciones tienen lugar durante 960 días de producción, lo que representa 4 años, tras los cuales de determina el coste promedio total para cada estrategia. Hemos considerado un tiempo de warm-up de 960, en el que no se registra ningún valor de coste. Este tiempo de warm-up, es necesario para evitar el efecto que alguna de las condiciones iniciales, en particular el stock inicial, puede tener sobre los resultados de la simulación. En (Vidal-carreras et al. 2009), los autores demostraron que un periodo de entre 600 y 1000 días de warm-up es adecuado para obtener resultados estables con algunas de las heurísticas más comúnmente empleadas para el ELSP. De cada experimento se han realizado 10 réplicas, tomando los datos promedio para cada uno de ellos.

\section{Resultados, análisis y discusión}

El objetivo del estudio de simulación es determinar cómo afecta el entorno de complejidad e incertidumbre al rendimiento de las diferentes estrategias analizadas. La medida de rendimiento empleada es el coste total, calculado como la suma de los costes de setup, y los costes de almacenamiento. Algunos autores (Vaughan 2007) emplean el inventario agregado como medida de rendimiento, pero por lo general, los estudios de simulación (Soman n.d.; Brander et al. 2005; Levén \& Segerstedt 2007) emplean el coste como media de rendimiento.

\subsection{Efecto del nivel de utilización}

La figura 1 muestra el comportamiento de las cinco estrategias descritas, ante ratios de utilización crecientes, para a) 10 artículos, y b) 40 artículos, y diferentes coeficientes de variación de la demanda. 

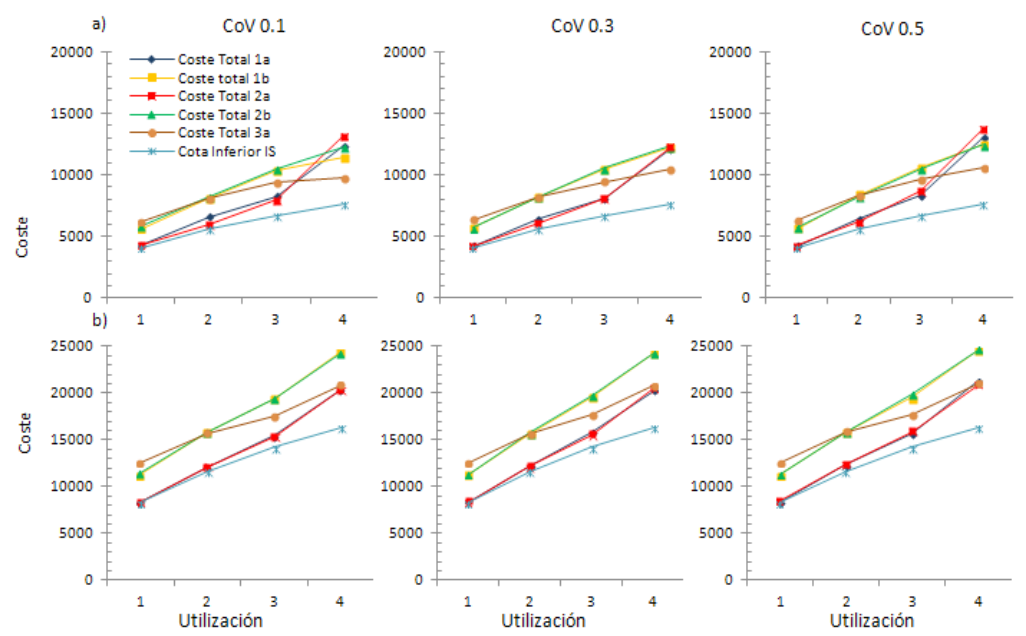

Fig. 1 Influencia del incremento del nivel de utilización sobre los costes.

a) 10 artículos, b) 40 artículos

En la figura se muestra también el comportamiento del coste para la solución teórica al modelo bajo el enfoque IS, que es una cota inferior. En relación con esto, es conocido (Bomberger 1966), que los costes tienen un crecimiento exponencial, en relación con la demanda, para el enfoque IS, de acuerdo con [4]

$$
T C=\sum_{i=1}^{g} \sqrt{2 A_{i} \boldsymbol{h}_{i} d_{i}\left(1-\frac{d_{i}}{p_{i}}\right)}
$$

No sólo se aprecia este incremento de costes, sino que este incremento tiene un patrón de comportamiento diferente para las diferentes estrategias empleadas. En particular, como se aprecia en la figura, las estrategias basadas en IS tienen un buen comportamiento a bajos niveles de utilización, lo cual es lógico porque se basan en la mejor solución para cada artículo. En cambio a mayores niveles de utilización esta solución empieza a ser difícilmente implementable. Esto provoca mayor número de roturas de stock y un mayor stock de seguridad para evitar que se produzcan, lo que hace mucho más convenientes otro tipo de estrategias, como las basadas en CC o la basada en la regla de Magee. La figura 2 ilustra los niveles de inventarios para el último año de simulación (1920 horas) para la estrategia $2 a$, en el caso de 10 artículos y utilización 0.88. Como se aprecia en la figura, esta estrategia de producción, con lotificación basada en IS, presenta graves roturas de stock a este nivel de utilización, lo que tiene un efecto directo sobre los costes totales.

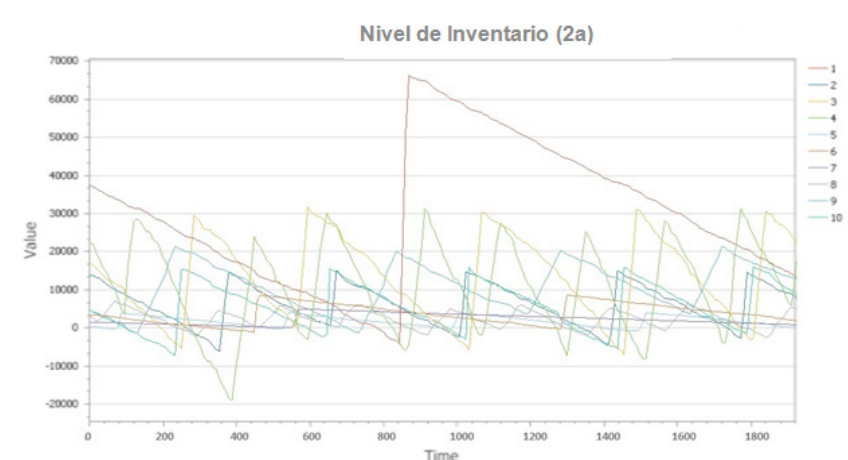

Fig. 2 Niveles de inventario por artículo. Estrategia 2a. 10 artículos. Utilización 0.88

\subsection{Efecto del número de artículos}

Los resultados en relación con el incremento del número de artículos, se muestran en la figura 3, para a) 10 artículos y b) 40 artículos. De forma similar a como ocurría con el incremento del factor de utilización, ante incremento del número de artículos, el coste total también se incrementa según [5]:

$$
C T_{g}=C T \sqrt{\frac{g \cdot p-d}{p-d}}
$$


La diferencia entre las estrategias 1a y $2 a$, basadas en IS, respecto a la cota inferior, aumenta con el nivel de utilización. Para un nivel de utilización 0.88 , la diferencia se hace tan grande que hace que sea más conveniente el empleo de estrategias basadas en CC. Es de destacar el buen comportamiento de las estrategias basada en la regla de Magee ante el incremento del número de artículos. Por tanto, mantener una estrategia de producción basada en IS, que para pocos artículos tiene un rendimiento aceptable, sería un error para un mayor número de artículos. Así pues, como se sugiere en Winands et al. (2011), es fundamental identificar en qué situación una determinada estrategia es efectiva. De la comparación de las cinco estrategas planteadas en los diferentes escenarios, se desprende la necesidad de profundizar en este estudio como herramienta de validación de las estrategias de resolución para el ELSP, en función del entorno productivo en el que van a ser utilizadas.
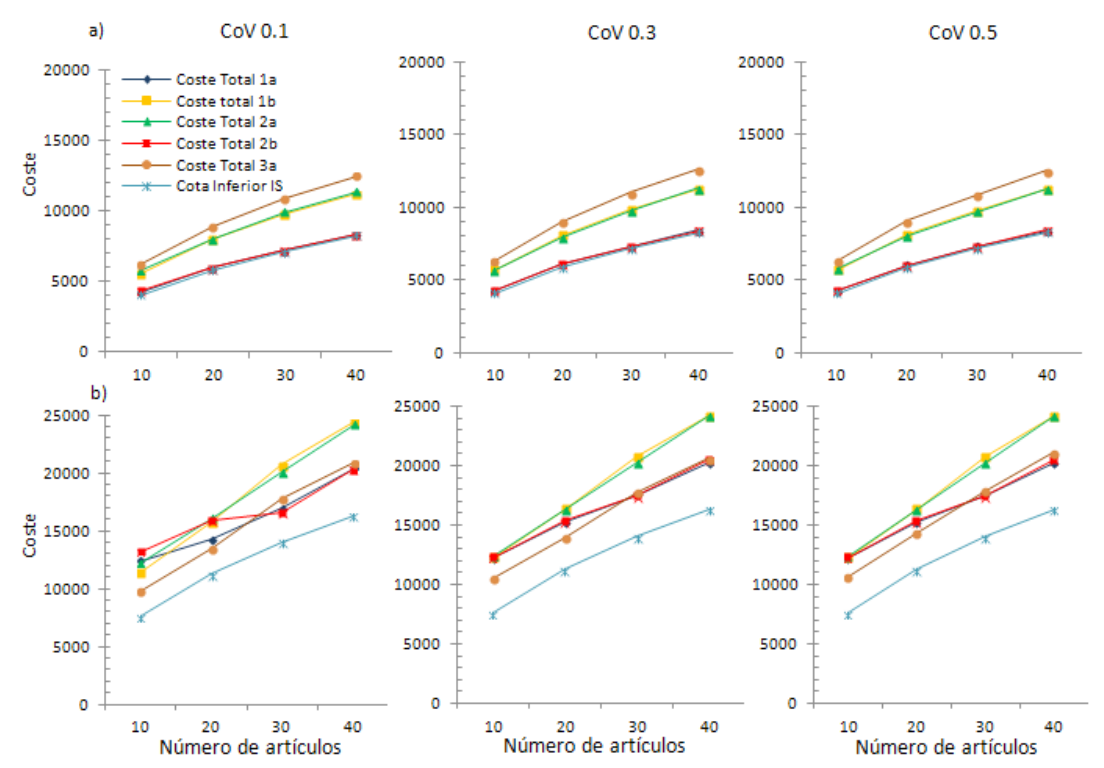

Fig. 3 Influencia del incremento del número de artículos sobre los costes.

a) Utilización 0.22 b) Utilización 0.88

\section{Conclusiones y líneas de trabajo futuro}

A partir de los resultados de este estudio, hemos probado que el rendimiento de una estrategia de producción para el ELSP se ve claramente afectado por el entorno productivo bajo el que es empleada. Se ha demostrado que el hecho de que una estrategia de producción obtenga un menor coste total de una determinada situación, no garantiza que la misma estrategia siga siendo mejor en otras condiciones específicas del mismo problema. Esta conclusión es absolutamente relevante para aplicaciones industriales en los problemas relacionados con el ELSP, donde las condiciones de contorno sufren variaciones sustanciales con mucha frecuencia, incluso en la misma instalación, por ejemplo en condiciones de demanda estacional. Como consecuencia de este resultado se desprende que las conclusiones de un estudio realizado utilizando un conjunto de datos específicos no pueden aplicarse con garantías, o incluso pueden provocar resultados contrarios a los esperados, cuando se aplica en un entorno diferente. En esta línea, se pretende ampliar este estudio de dos líneas de trabajo. Por un lado, pretendemos demostrar que este análisis presenta resultados similares al aplicarse a otras estrategias de producción presentadas en la literatura, cuyo rendimiento ha sido analizado en relación con otras estrategias, pero no de acuerdo con la complejidad del escenario en estudio. Por otro lado, se pretende introducir otros drivers de complejidad e incertidumbre en el análisis, como los mencionados en la introducción. En este sentido, nuevas variantes del problema objeto de estudio (diferentes problemas marco) introducen nuevos drivers de complejidad. Otras variantes del problema que introducen nuevos drivers de complejidad son, por ejemplo, la consideración de estacionalidad de la demanda, la existencia de tiempos de setup dependientes de la secuencia, o ratios de producción variables, entre otros. En cuanto a la incertidumbre, y como se mencionó en la introducción , teniendo en cuenta el ELSP clásico, aparte de la demanda, los tiempos de proceso o los tiempos de setup , pueden mostrar un comportamiento estocástico, lo que introduce incertidumbre en el problema . En consecuencia, las consideraciones de averías o la disponibilidad de las existencias introducirían otros drivers 
de incertidumbre, bajo los cuales pretendemos ampliar el análisis.

Por último, en la medida en que los resultados de este trabajo se derivan de un estudio de simulación, resulta de interés para los autores evaluar la influencia de algunos de los factores experimentales sobre las conclusiones del estudio. Entre los factores experimentales cuya influencia se pretende analizar y que, en nuestra opinión, no han recibido suficiente atención en la literatura se encuentran la duración del experimento, los inventarios iniciales, el periodo de warm-up, y el número de replicas por experimento en relación con el intervalo de confianza.

\section{Referencias}

ALLAHVERDI, A. ET AL., (2008). "A survey of scheduling problems with setup times or costs". European Journal of Operational Research, 187(3), pp.9851032.

BOMBERGER, E.E., (1966). "A Dynamic Programming Approach to a Lot Size Scheduling Problem". Management Science, 12(11), pp.778-784.

BRANDER, P. \& FORSBERG, R., (2006). "Determination of safety stocks for cyclic schedules with stochastic demands". International Journal of Production Economics, 104(2), pp.271-295.

BRANDER, P., LEVÉN, E. \& SEGERSTEDt, A., (2005). "Lot sizes in a capacity constrained facility-a simulation study of stationary stochastic demand". International Journal of Production Economics, 93-94, pp.375-386.

CHAN, H.K., HO CHUNG, S. \& LIM, M.K., (2013). "Recent research trend of economic lot scheduling problems". S. Shaaban, ed. Journal of Manufacturing Technology Management, 24(3), pp.465-482.

DOBSON, G., (1987). "Economic lot-scheduling problem: achieving feasibility using time-varying lot sizes". Operations Research, 35(5), pp.764-771.

FRIZELLE, G.D.M., (1996). "Getting e measure of coimplexity". Manufacturing Engineer, 75(6), pp.268-270.

HANSSMANN, F., (1962). Operations Research in Production and Inventory Control, New York: John Wiley and Sons.
HARRIS, F.W., (1913). "How Many Parts to Make at Once". The magazine of Management, 10(2), pp.135-6-152.

HSU, W.L., (1983). "On the general feasibility test of scheduling lot sizes for several products on one machine". Management Science, 29(1), pp.93-105.

KOU, Y. \& YANG, J.J., (2009). "A Simulation-Based Methodology for Lot-Size Optimization". Key Engineering Materials, 392-394, pp.866-872.

LEACHMAN, R.C. \& GASCON, A., (1988). "A Heuristic Scheduling Policy for Multi-Item, Single-Machine Production Systems with Time-Varying, Stochastic Demands". Management Science, 34(3), pp.377390.

LEVÉN, E. \& SEGERSTEDT, A., (2007). "A scheduling policy for adjusting economic lot quantities to a feasible solution". European Journal of Operational Research, 179(2), pp.414-423.

LÖHNDORF, N., RIEL, M. \& MINNER, S., (2014). "Simulation optimization for the stochastic economic lot scheduling problem with sequence-dependent setup times". International Journal of Production Economics, 157, pp.170-176.

MAGEE, J.. \& BOODMAN, D.M., (1967). Production Planning And Inventory Control, New York: McGraw-Hill.

SEGERSTEDT, A., (1999). "Lot sizes in a capacity constrained facility with available initial inventories". International Journal of Production Economics, 59(1-3), pp.469-475.

SOMAN, C.A., Make-to-order and make-to-stock in food processing industries.

SOMAN, C.A., PIETER VAN DONK, D. \& GAALMAN, G., (2006). "Comparison of dynamic scheduling policies for hybrid make-to-order and make-to-stock production systems with stochastic demand". International Journal of Production Economics, 104(2), pp.441-453.

SOX, C.R. et al., (1999). "A review of the stochastic lot scheduling problem". International Journal of Production Economics, 62(3), pp.181-200.

VAUGHAN, T.S., (2007). "Cyclical schedules vs. dynamic sequencing: Replenishment dynamics and inventory efficiency". International Journal of Production Economics, 107(2), pp.518-527. 
VERGIN, R.C. \& Lee, T.N., (1978). Scheduling Rules For The Multiple Product. , 16(1), pp.64-74.

VIDAL-CARRERAS, P.I. et al., (2009). Estabilidad del horizonte de programación en la aplicación de heurísticas al problema del ELSP con Coproducción Deliberada.
WINANDS, E.M.M., ADAN, I.J.B.F. \& VAN HOUTUM, G.J., (2011). "The stochastic economic lot scheduling problem: A survey". European Journal of Operational Research, 210(1), pp.1-9.

YAO, M.J. \& HUANG, J.X.,(2005). "Solving the economic lot scheduling problem with deteriorating items using genetic algorithms". Journal of Food Engineering, 70, pp.309-322. 